\title{
Differential Antibody Response of Cattle Immunized with Anaplasma marginale Derived from Bovine Erythrocytes or Cultured Tick Cells
}

\author{
Jose C. Garcia-Garcia*, José de la Fuente*, Edmour F. Blouin*, Jeremiah T. Saliki*, Katherine M.
} Kocan*

Department of Veterinary Pathobiology, College of Veterinary Medicine, Oklahoma State University, Stillwater, OK 74078, USA

Anaplasmosis is a hemolytic disease of cattle caused by the rickettsial tick-borne pathogen Anaplasma marginale (Rickettsiales: Anaplasmataceae). Killed vaccines have been used for the control of anaplasmosis in the United States. These previous vaccines used A. marginale antigen harvested from infected bovine erythrocytes and bore the risk of being contaminated with bovine cells and other pathogens. Recently, a cell culture system was developed for the propagation of $A$. marginale using a continuous cell line (IDE8) that was originally derived from embryos of Ixodes scapularis. The six major surface proteins (MSPs) described on the erythrocytic stage of $A$. marginale were found to be conserved on cell culture-derived organisms. A. marginale antigen derived from cell culture was tested as an immunogen for cattle and the cell culture-derived vaccine prevented clinical anaplasmosis in vaccinated cattle. In this research we report the differential antibody response of cattle to erythrocyte- and cell culture-derived antigen that was demonstrated in three independent vaccine trials. Cattle immunized with $A$. marginale from tick cells or bovine erythrocytes produced antibodies against the $A$. marginale MSP5 but a differential antibody response to MSP1a and MSP1b was observed. Cattle immunized with erythrocyte-derived A. marginale elicited an antibody response mainly against MSP1a, while animals immunized with cell culturederived antigen produced predominantly antibodies to MSP1b. The molecular basis of this differential antibody response was then studied by comparing the amounts of MSP1a, MSP1b and MSP5 on A. marginale harvested from the two host cells. The amount of MSP1b and MSP5 was similar on A. marginale from both host cells, but the amount of MSP1a was higher in the erythrocyte-derived $A$. marginale. These results were further demonstrated using confocal microscopy (Fig. 1). These differences could be regulated at transcriptional, translational or posttranslational levels. Since MSP1a has been shown to be an A. marginale adhesin for tick cells, biological transmission of the pathogen could be enhanced by increased levels of this surface protein. Differences in the level of surface exposed molecules also may contribute to phenotypic and antigenic variation in the pathogen. 


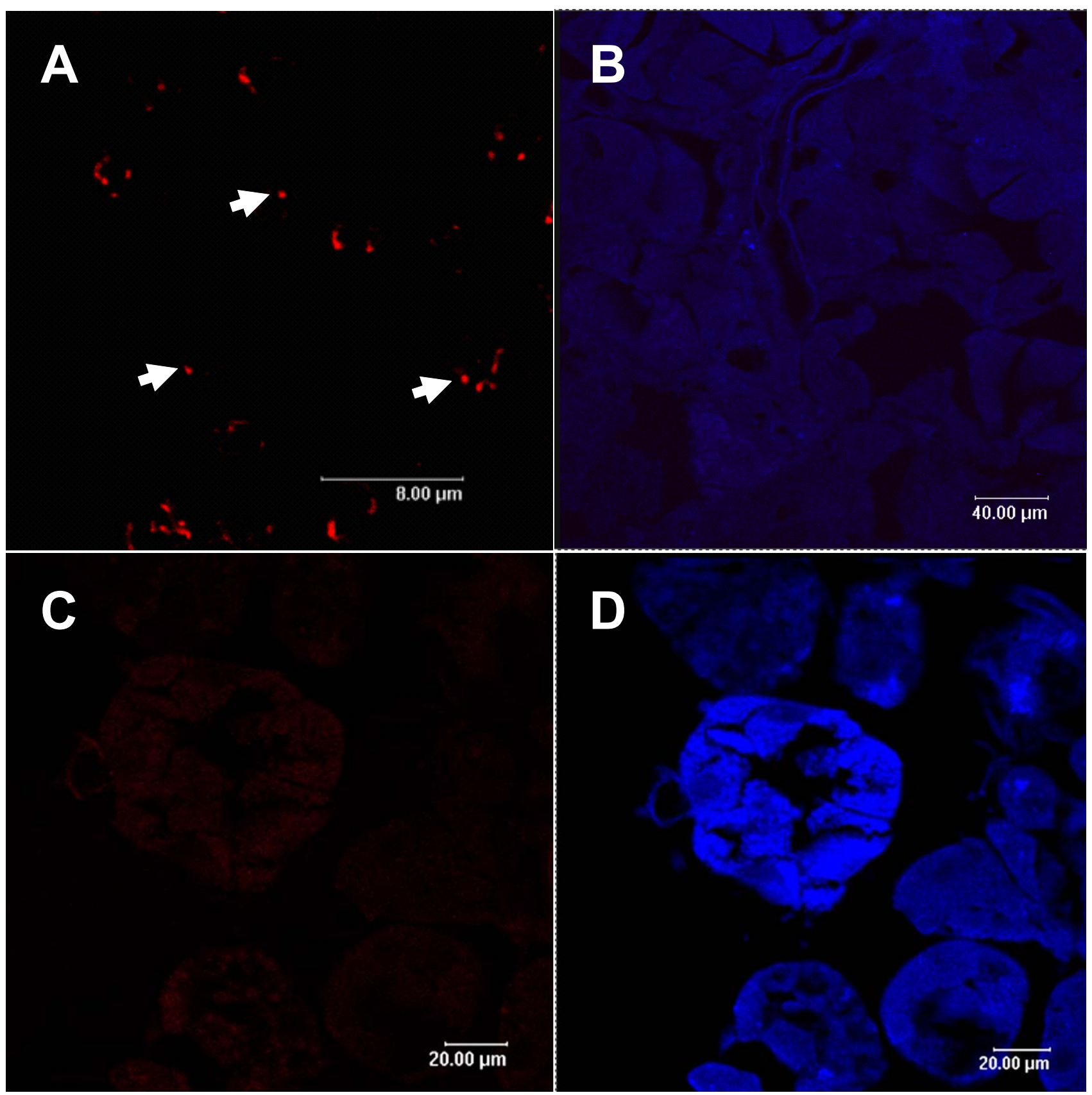

Figure 1. Confocal microscopy of (A) a smear of $A$. marginale infected bovine erythrocytes, (B) a cross-section of uninfected $D$. variabilis salivary glands and (C,D) a cross-section of $A$. marginale infected D. variabilis salivary glands, probed with (A,C) MAb ANA15D2 (anti-MSP1a) or (B, D) MAb ANAF16C1 (anti-MSP5), labeled with Alexa Fluor 546 and Alexa Fluor 633, respectively. Arrows indicate expression of MSP1a in the A. marginale inclusion bodies in bovine erythrocytes. Figures $C$ and D correspond to a cross-section of infected salivary gland that was simultaneously incubated with labeled anti-MSP1a and anti-MSP5 MAbs and examined for the presence of both labels. 